\title{
An Early Decision Algorithm to Accelerate Web Content Filtering
}

\author{
Ying-Dar Lin ${ }^{1}$, Po-Ching Lin ${ }^{1}$, Ming-Dao Liu ${ }^{1}$ and Yuan-Cheng Lai ${ }^{2}$ \\ ${ }^{1}$ Department of Computer Science \\ National Chiao Tung University, HsinChu, Taiwan \\ Email: \{ydlin, pclin, moliu\}@cis.nctu.edu.tw \\ ${ }^{2}$ Department of Information Management \\ National Taiwan University of Science and Technology, Taipei, Taiwan \\ Email: laiycecs.ntust.edu.tw
}

\begin{abstract}
Real-time content analysis can be a bottleneck in Web filtering. This work presents a simple, but effective early decision algorithm to accelerate the filtering process by examining only part of the Web content. The algorithm can make the filtering decision, either to block or to pass the Web content, as soon as it is confident with a high probability that the content should belong to a banned or an allowable category. The experiments show the algorithms can examine only around one-fourth of the Web content on average, while the accuracy remains fairly good: $89 \%$ in the banned content and $93 \%$ in the allowable content. This algorithm can complement other Web filtering approaches to filter the Web content with high efficiency.
\end{abstract}

\section{Introduction}

Massive volume of Internet content is widely accessible nowadays. One can easily view improper content at will without access control. For example, an employee may watch stock information during office hours. Web filtering products can enforce the access control. The up-to-date products have widely adopted content analysis besides the URL-based approach [1]. Content analysis works with the URL-based approach to relieve the efforts of maintaining the URL list and to reduce the number of false negatives. The analysis classifies the Web content to a certain category first, and makes the filtering decision, either to block or to pass the content.

Despite the ongoing research on image and video content classification, text classification is typically the most efficient approach to Web content analysis. Many text classification algorithms have been around with high accuracy. They are often assumed to run off-line, so their execution time is rarely discussed. However, the efficiency of these algorithms is critical because slow content analysis in Web filtering incurs long user response time. The issues of accelerating the analysis should deserve attention. 
This work presents a simple, but effective early decision algorithm to accelerate the filtering from the observation that the filtering decision can be made before scanning the entire content, as soon as the content can be classified into a certain category. A fast decision is particularly important since most Web content is normally allowable and should pass the filter as soon as possible.

The rest of this paper is organized as follows. Section 2 provides the background of this work. The early decision algorithm is described in Section 3. Section 4 exhibits the accuracy and efficiency of this algorithm from the experimental results and discusses the deployment issues in a practical environment. Finally, Section 5 concludes this work.

\section{Background}

Yang et al. and Sebastiani [2], [3] gave a comprehensive survey of existing text classification algorithms. These algorithms are shown to achieve around $80 \%$ of accuracy or higher, measured by the average of recall and precision. Recall is defined to be the ratio of the number of correct positive predictions divided by the number of positive examples, while precision is the ratio of the number of correct positive predictions divided by the number of positive predictions. Among these algorithms, we choose Naïve Bayesian classification as the base of the early decision algorithm for its simplicity. Other classification algorithms can follow the principle to accelerate the classification.

The Bayesian classification is divided into two stages: training and classification. The training stage learns the probabilistic parameters of the generative model from a set of training documents, $\mathrm{D}=\left\{d_{1}, \ldots \ldots, d_{|D|}\right\}$. Each document consists of a sequence of words from a vocabulary set $\mathrm{V}=\left\{w_{1}, w_{2}, \ldots w_{|V|}\right\}$ and has been labeled with some category from a set of categories $\mathrm{C}=\left\{c_{1}, c_{2}, \ldots, c_{|c|}\right\}$ before the training. Two types of parameters are included in the model: (1) $\mathrm{P}\left(w_{t} \mid c_{j}\right)$ : the estimated probability of word $w_{t}$ given category $c_{j}$ and (2) $\mathrm{P}\left(c_{j}\right)$ : the estimated probability of category $c_{j}$. These parameters are derived by [4]

$$
P\left(w_{t} \mid c_{j}\right)=\frac{1+\sum_{i=1}^{|D|} N\left(w_{t}, d_{i} \mid d_{i} \in c_{j}\right)}{|V|+\sum_{t=1}^{|V|} \sum_{i=1}^{|D|} N\left(w_{t}, d_{i} \mid d_{i} \in c_{j}\right)},
$$

where $\mathrm{N}\left(w_{t}, d_{i}\right)$ is the times word $w_{t}$ appears in document $d_{i}$, and

$$
P\left(c_{j}\right)=\frac{1+\sum_{i=1}^{|D|} P\left(d_{i} \in c_{j}\right)}{|C|+|D|} .
$$


In the classification stage, the posterior probability $\mathrm{P}\left(c_{j} \mid d_{i}\right)$ with which a test document $d_{i}$ belongs to category $c_{j}$ is derived. The category $c_{j}$ that maximizes $\mathrm{P}\left(c_{j} \mid d_{i}\right)$ is the one that $d_{i}$ belongs to. $\mathrm{P}\left(c_{j} \mid d_{i}\right)$ is derived by

$$
P\left(c_{j} \mid d_{i}\right)=\frac{P\left(c_{j}\right) P\left(d_{i} \mid c_{j}\right)}{P\left(d_{i}\right)}=\frac{P\left(c_{j}\right) \prod_{k=1}^{d i} P\left(w_{d i, k} \mid c_{j}\right)}{P\left(d_{i}\right)}
$$

where $w_{d i, k}$ is the $k$-th word in document $d_{i}$. Notice that the document $d_{i}$ is viewed as an ordered sequence $\left\langle w_{d i, 1}, w_{d i, 2}, \ldots, w_{d i,|d i|}\right\rangle$, with the assumption that the probability of a word occurrence is independent of its position in the document, given the document category $c_{j}$, so that $\mathrm{P}\left(d_{i} \mid c_{j}\right)$ can be written as the product of individual probabilities $\mathrm{P}\left(w_{d i, k} \mid c_{j}\right)$.

\section{The Early Decision Algorithm}

The philosophy behind the early decision algorithm is to make the filtering decision from the front partial Web content. Fig. 1 presents the average keyword distribution of both banned and allowable Web pages in our investigation. The keyword position is normalized by the page length. The keywords in almost all Web pages tend to be distributed uniformly throughout the content or appear more in the front part according to this investigation. The Web content in a banned category starts to exhibit much more keywords than that in an allowable category since the front part. In other words, keywords from the front partial content can reveal the category of the Web content and serve as the clues to filtering.

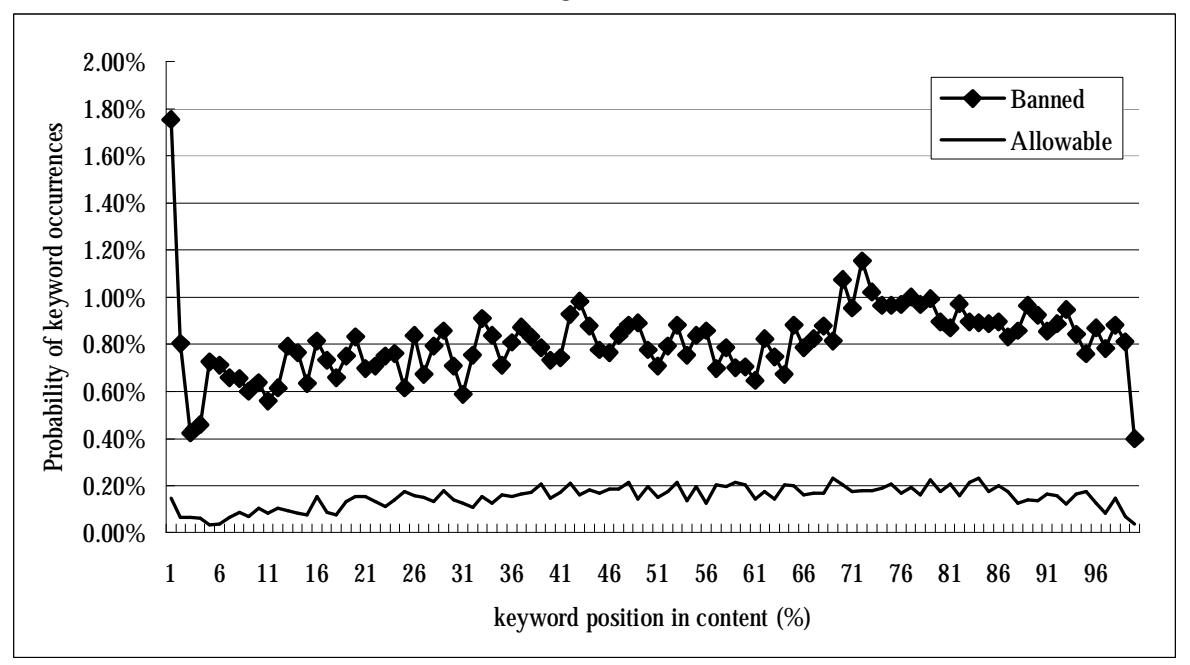

Fig. 1. The distribution of keyword positions in typical Web pages 
Like the Bayesian classification, the filtering engine is trained off-line from the Web content in the banned categories. The Bow library and its front-end, Rainbow [5 perform the training herein, extracting keywords as the features from the target categories. The keywords with the information gains larger than a threshold are selected. Stop words, such as "the", "of" and so on, should be dropped because they help little in classification. The words inside the HTML tags are also ignored so that a malicious user cannot stuff unrelated content in the tags, particular in the front part of the Web page, to deceive the filter. If the malicious user fills the Web text outside the tags with irrelevant content to confuse the filter, the irrelevant content will be displayed in the browser and will spoil the layout of the Web pages - a great limitation on the design of the Web pages.

The score of keyword $w_{t}$ that should belong to a category $c_{j}$ is defined to be $\log \mathrm{P}\left(w_{t} \mid c_{j}\right)$, which can be derived in the training stage. Taking the logarithm simplifies the computation of the posterior probability $\mathrm{P}\left(c_{j} \mid d_{i}\right)$ from multiplication operations to score accumulation with independence assumption between words [4]. The scores are accumulated while the content is scanned from the front to the end.

In the filtering stage, given $n \%$ of the content that has been scanned and the score $m$ or less that has been accumulated, the probability that the content should belong to a category $c$ is derived from

$$
P(c \mid D(n, m))=\frac{P(D(n, m) \mid c) P(c)}{P(D(n, m) \mid c) P(c)+P\left(D(n, m) \mid c^{\prime}\right) P\left(c^{\prime}\right)} .
$$

1. $D(n, m)$ : the event that the filter has read $\mathrm{n} \%$ of the content and has observed the score accumulation $m$ or less.

2. $P(c)$ : the estimated probability that category c appears in typical Web content.

3. $P\left(c^{\prime}\right)$ : the estimated probability that category c does not appear in typical Web content. $P\left(c^{\prime}\right)=1-P(c)$.

4. $\quad P(D(n, m) \mid c)$ : the estimated probability that $D(n, m)$ happens given that the content belongs to category c. The estimate of $P(D(n, m) \mid c)$ is the number of Web pages in $c$ that $D(n, m)$ happens divided by the number of Web pages in c.

5. $\quad P\left(D(n, m) \mid c^{\prime}\right)$ : defined similarly as $P(D(n, m) \mid c)$, except that $c$ is replaced with $c^{\prime}$.

In the training phase, two two-dimensional indexed tables of $P\left(D(n, m) \mid c_{i}\right)$ and $P\left(D(n, m) \mid c_{i}\right)$ are built for each $n$ and $m$ from the training examples, where $c_{i} \in \mathrm{C}$. The values of $P\left(c_{i}\right)$ and $P\left(c_{i}{ }^{\prime}\right)$ can be estimated beforehand or dynamically tuned in a running environment by recording and analyzing actual Web content. Fig. 2 presents the early decision algorithm. Two thresholds, $\mathrm{T}_{\text {bypass }}$ and $\mathrm{T}_{\text {block }}$, are defined to be 0.1 and 0.9 herein. $P C D_{i}$ is the estimate that the content should belong to a category $c_{i}$. If $P C D_{i}$ is less than $\mathrm{T}_{\text {bypass }}$ for all $c_{i}$ in the list of banned categories, this means the content is unlikely to be banned and the remaining content should be bypassed. In contrast, if there exists some $c_{i}$ in the list of banned categories such that $P C D_{i}$ is 


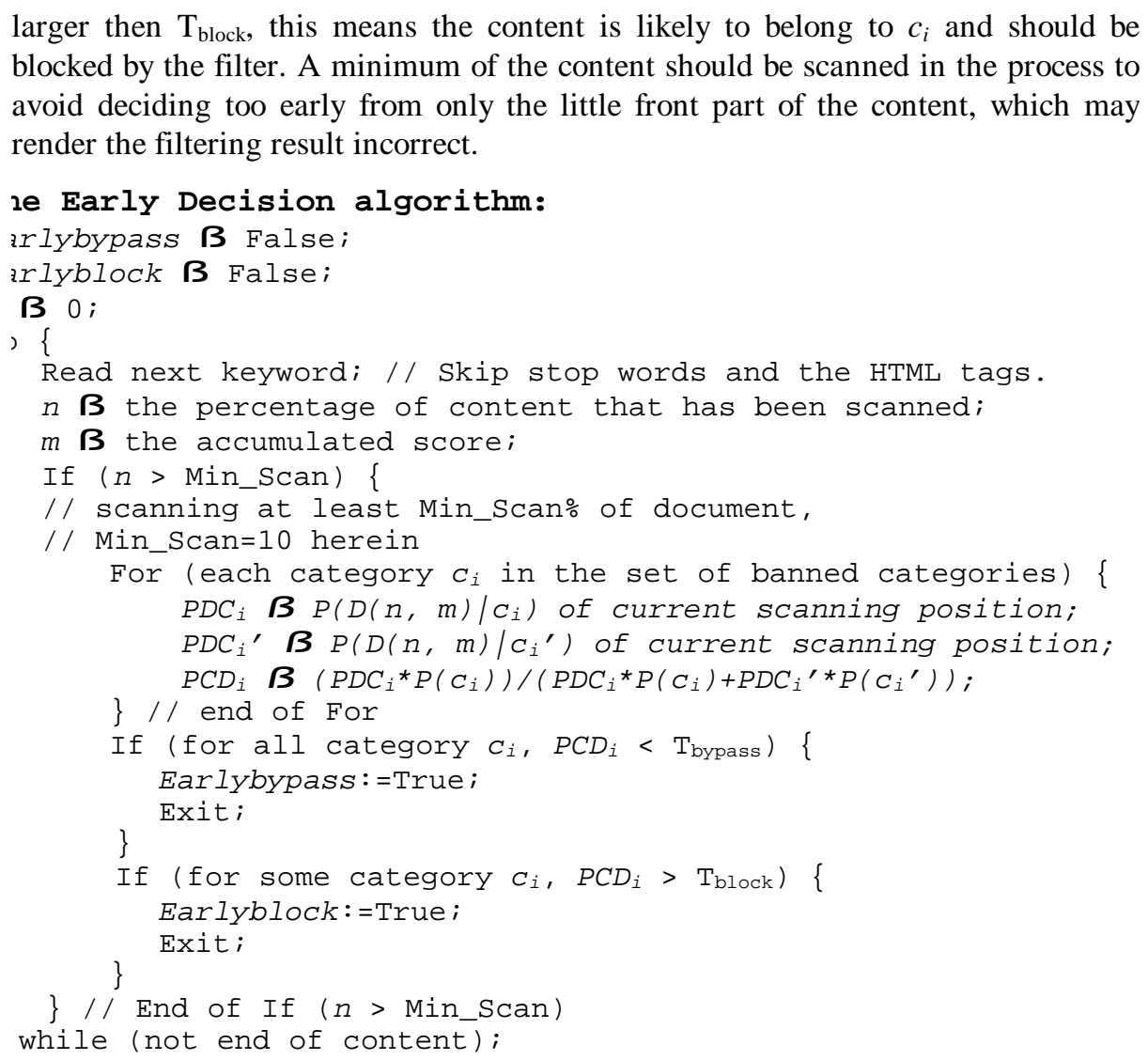
blocked by the filter. A minimum of the content should be scanned in the process to avoid deciding too early from only the little front part of the content, which may render the filtering result incorrect.

g. 2. The pseudo code of the early decision algorithm

\section{Experiments}

\subsection{Performance metrics}

The F1 measure, initially introduced by Van Rijsbergen [6], takes the harmonic average of the recall and the precision as the measure of accuracy. To measure the acceleration, the average scan ratio and the average throughput are defined by

$$
\text { Average scan ratio }=\frac{\text { Total bytes scanned }}{\text { Total bytes in the content }},
$$




$$
\text { Averagethroughput }=\frac{\text { Total bits in the content being filtered }}{\text { Total execution time of the filtering }(\mathrm{sec})}
$$

\subsection{Experimental results and discussion}

Totally $300 \mathrm{Web}$ pages are randomly collected from the YAHOO directory services [7] for the experiment in four typically banned categories: Pornography, Game, Online-Shopping and Finance. Another 300 pages are also randomly collected from other categories as the allowable content. The extracted keywords in the training stage are searched through the Web content with a multiple string matching algorithm. Since short patterns are not uncommon in natural languages, a sub-linear time algorithm, such as the Wu-Manber algorithm [8], can hardly take any advantages. The filtering algorithm is implemented with Lex [9], which is based on the Aho-Corasick algorithm [10], so the performance is less sensitive to short patterns.

The accuracy of the original Bayesian classifier, which scans the entire content, is compared with that of the early decision algorithm for the four banned categories in Table 1. Only the shopping category suffers noticeable accuracy degradation whereas the other categories remain fairly good accuracy. A careful examination reveals it is because the keywords in the shopping category include many ambiguous words that also appear in allowable content. If this is the case, more other examples from the category can be trained until better keywords that lead to higher accuracy are derived The filtering accuracy by averaging the accuracy of the four banned categories and that of allowable content are presented in Table 2. The filtering accuracy of both types of content with the early decision keeps fairly close to that when scanning the entire content, but only $17.22 \%$ of content in the banned categories and $26.51 \%$ in the allowable categories on average are scanned. This means a large portion of the Web content can be bypassed in Web filtering, and the execution time can be significantly shorter.

Table 1. Comparisons of classification accuracy

\begin{tabular}{|c|c|c|c|c|}
\hline $\begin{array}{l}\text { Algo } \\
\text { Cate }\end{array}$ & Porn & Game & Shopping & nce \\
\hline $\begin{array}{ll}\text { Original } & \text { Bayesian } \\
\text { classifier } & \\
\end{array}$ & $\begin{array}{c}\text { Pr Re } \mathrm{F} 1 \\
1.00 .993 .996\end{array}$ & $\begin{array}{|ccc|}\operatorname{Pr} & \operatorname{Re} & \mathrm{F} 1 \\
1.00 & .971 & .985\end{array}$ & $\begin{array}{rll}\operatorname{Pr} & \operatorname{Re} & \mathrm{F} 1 \\
1.00 & .975 & .987\end{array}$ & $\begin{array}{rrr}\operatorname{Pr} & \operatorname{Re} & \mathrm{F} 1 \\
.896 & 1.00 & .945 \\
\end{array}$ \\
\hline Early decision & .977 .918 .947 & .958 .819 .883 & .866 .750 .804 & .9640 .90 .931 \\
\hline
\end{tabular}

Table 2. Comparisons of filtering accuracy and average scan ratio

\begin{tabular}{|l|c|c|c|c|}
\hline $\begin{array}{l}\text { Algorithm/ } \\
\text { Category types }\end{array}$ & Banned & Allowable & $\begin{array}{c}\text { Average scan } \\
\text { ratio in banned } \\
\text { content }\end{array}$ & $\begin{array}{c}\text { Average scan } \\
\text { ratio in allow- } \\
\text { able content }\end{array}$ \\
\hline Early decision & $\begin{array}{c}\operatorname{Pr} \text { Re F1 } \\
.941 .847 .892\end{array}$ & $\begin{array}{c}\text { Pr Re F1 } \\
.947 .920 .934\end{array}$ & $17.22 \%$ & $26.51 \%$ \\
\hline
\end{tabular}


False positives of allowable traffic are usually unacceptable in a practical environment and a higher threshold $\mathrm{T}_{\text {block }}$ would be better. By lifting the threshold $\mathrm{T}_{\text {block }}$ to 1.0 , false positives in the allowable categories can be almost avoided. Table 3 presents that a higher threshold also results in more false negatives in the banned categories because some banned content cannot reach such a high threshold. Choosing a proper threshold is a tradeoff in a practical environment.

The execution time and throughput of the original Bayesian classifier and the early decision algorithm are compared on a PC with Intel Pentium III $700 \mathrm{MHz}$ and $64 \mathrm{MB}$ of RAM. Table 4 presents the average execution time and the throughput of filtering the banned and allowable content. The results show significant improvement in throughput, about five times higher than that of the original Bayesian classifier for banned content and nearly four times higher for allowable content.

Table 3. Accuracy in the setting of no false positives in allowable content

\begin{tabular}{|c|c|c|c|c|}
\hline Setting & Porn & Game & Shopping & Finance \\
\hline $\begin{array}{l}\text { Original Bayesian } \\
\text { classifier }\end{array}$ & \begin{tabular}{|cc}
$\operatorname{Pr}$ & $\operatorname{Re} \mathrm{F} 1$ \\
1.00 & .993 .996
\end{tabular} & \begin{tabular}{|ccc}
$\operatorname{Pr}$ & $\operatorname{Re}$ & $\mathrm{F} 1$ \\
1.00 & .971 & .985
\end{tabular} & $\begin{array}{ccc}\operatorname{Pr} & \operatorname{Re} & \mathrm{F} 1 \\
1.00 & .975 & .987\end{array}$ & $\begin{array}{ccc}\operatorname{Pr} & \operatorname{Re} & \mathrm{F} 1 \\
.896 & 1.00 & .945\end{array}$ \\
\hline No false positives & 1.00 .773 .871 & 1.00 .623 .767 & $\begin{array}{lll}1.00 & .55 .709\end{array}$ & 1.00 .730 .843 \\
\hline
\end{tabular}

Table 4. Comparison of the throughput of the early decision algorithm and the original Bayesian classifier

\begin{tabular}{|l|c|c|}
\hline \multicolumn{1}{|c|}{ Algorithm } & $\begin{array}{c}\text { Execution time } \\
(\mu \mathrm{s})\end{array}$ & $\begin{array}{c}\text { Throughput } \\
(\mathrm{Mb} / \mathrm{s})\end{array}$ \\
\hline $\begin{array}{l}\text { Original Bayesian clas- } \\
\text { sifier }\end{array}$ & 1333772 & 41.05 \\
\hline $\begin{array}{l}\text { Early decision for } \\
\text { banned content }\end{array}$ & 241887 & 226.36 \\
\hline $\begin{array}{l}\text { Early decision for al- } \\
\text { lowable content }\end{array}$ & 239895 & 156.68 \\
\hline
\end{tabular}

Many commercial products and open source packages in our investigation, such as DansGuardian [11], can block a page as the score accumulation achieves the given threshold configured arbitrarily by the users. The early decision algorithm compares the threshold with the probability estimation of the classification, rather than the score itself. The advantages of the early decision algorithm over the method in DansGuardian are two points. (1) The two parameters, $\mathrm{T}_{\text {bypass }}$ and $\mathrm{T}_{\text {block}}$, have stronger association with the accuracy than the threshold of score in DansGuardian. The filtering can then be better tuned directly according to the desired accuracy. The choice of a proper threshold of score in DansGuardian to get the desired accuracy needs to take more efforts by trial and error. (2) The early decision algorithm accelerates not only filtering blocked Web pages, but also filtering allowable pages. The acceleration is particularly significant when the Web accesses are mostly allowable content. 
The early decision algorithm is also implemented by modifying the filtering code in DansGuardian. The throughput is enhanced by about three times on average than that in DansGuardian in our testing samples because of the acceleration from the allowable content and the better criterion to decide the blocking. This algorithm can also be implemented into other Web filtering products to accelerate the filtering process.

\subsection{Practical considerations in deployment}

With the increasing number of categories to be classified, ambiguity between these categories may increase. In our opinion, the proper place to perform Web content filtering is restricted to the edge devices for performance reason. Such edge devices usually require fewer banned categories. The problem with increasing number of categories is not that serious.

The early decision algorithm is supposed to complement other Web filtering approaches, such as URL filtering, not to replace them. Some situations, such as SSL connections and content of images, video, Flash objects or Java applets, are nontrivial to analyze on line. This algorithm can work with other approaches to filter the Web content with high efficiency.

The two thresholds, $\mathrm{T}_{\text {bypass }}$ and $\mathrm{T}_{\text {block}}$, can be tuned according to the tradeoffs between accuracy and efficiency. The accuracy can be increased at the cost of less efficiency by decreasing $\mathrm{T}_{\text {bypass }}$ or increasing $\mathrm{T}_{\text {block}}$, and the efficiency can be increased at the cost of less accuracy by increasing $\mathrm{T}_{\text {bypass }}$ or decreasing $\mathrm{T}_{\text {block}}$. The tuning depends on which is more important for an organization: accuracy or efficiency.

\section{Conclusions}

This work addresses the problem of possibly long delay from text classification algorithms to perform run-time content analysis of Web content. An early decision algorithm to decide to either block or pass the content as soon as the decision can be made is presented. A significant performance improvement is observed. The throughput is increased by about five times higher for banned content and nearly four times higher for allowable content while the accuracy remains fairly good. In the F1 measure, the accuracy can achieve about $89 \%$ for filtering banned content, and about $93 \%$ for allowable content.

The early decision algorithm is simple but effective. The same rationale behind this algorithm can be applied to other content filtering applications as well, such as anti-spam. The algorithm can be also combined with more features other than keywords from the text to further increase the overall accuracy of the content filter. Besides, the filtering can be further accelerated by combining the URL-based method with the cached results. That is, by caching the URLs of the filtered Web pages, duplicate filtering on the same Web page can be avoided. Content analysis can be 
skipped if the cached URL is matched. The maintenance of the URL list is also facilitated.

\section{Acknowledgement}

This work was supported in part by the Taiwan Information Security Center (TWISC), National Science Council under the Grants NSC 94-3114-P-001-001-Y and NSC 94-3114-P-011-001.

\section{References}

1. Internet Filter Review 2005. Available at http://internet-filter-review.toptenreviews.com/

2. Y. Yang and X. Liu, "A re-examination of text categorization methods", Proc. of SIGIR'99, 22nd ACM International Conference on Research and Development in Information Retrieval (1999) 42-49

3. F. Sebastiani, "Machine learning in automated text categorization," ACM Computing Survey, vol. 34, No. 1 March (2002) 1-47

4. Tom Mitchell. Machine Learning, McGraw Hill (1996)

5. The Rainbow library. Available at http://www-2.cs.cmu.edu/ mccallum/bow/rainbow/

6. C.J.Rijsbergen. Information Retrieval, Butterworths, London (1979)

7. YAHOO directory services. Available at http://www.yahoo.com

8. S. Wu and U. Manber, "A fast algorithm for multi-pattern searching", Technical Report TR-94-17, University of Arizona (1994)

9. M. E. Lesk, "Lex - A lexical analyzer generator", Comp. Sci. Tech. Rep. No. 39. Bell Laboratories (1975)

10. Aho, A. V., and M. J. Corasick, "Efficient string matching: an aid to bibliographic search" Comm. of the ACM, 18 (1975) 333-340

11. The DansGuardian Web filtering tools. Available at http://dansguardian.org/ 